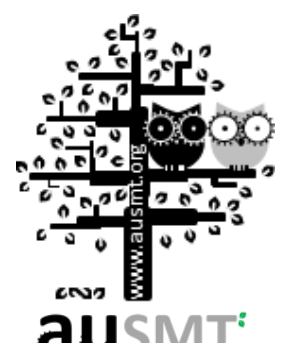

\title{
Analysis of Demand for Intelligent Automation in Taiwan's Notebook PC Assembly Industry
}

\section{Ying-Mei Tai*}

Industrial Economics and Knowledge Center (IEK), Industrial Technology Research Institute (ITRI), Taiwan

(Received 5 August 2013; Published on line 1 September 2013)

*Corresponding author: yyhuang@itri.org.tw

DOI: $10.5875 /$ ausmt.v3i3.218

Abstract: Assembly manufacturers of $3 \mathrm{C}$ (computers, communications and consumer electronics) products in Taiwan play a crucial role in the global market. Most of these manufacturer's plants, however, are located in China, where quality issues tend to be a problem. This study explores the notebook PC assembly industry, which is characterized by higher output values, growth rates and market share in the $3 \mathrm{C}$ assembly sector. The study aims to assess the potential demand for intelligent automation. Investments in intelligent automation must be based on potential input-output benefits, thus manufacturers can only create potential benefits through selecting key processes which are characterized by relatively high standardization and high quality thresholds.

Keywords: Intelligent automation; notebook PC assembly; mainland china

\section{Introduction}

Taiwan assembly manufacturers play an important role in the global market for 3C products. In 2011, their total output value was approximately 169.4 billion USD, accounting for $40 \%$ of global market share. In particular, Taiwan manufacturers accounted for more than $90 \%$ of global production of motherboards, notebook PCs and tablet PCs. While these firms are based in Taiwan, most of their manufacturing capacity is in mainland China, allowing them to meet demand for small-volume, large-variety production. In fact, $99 \%$ of notebook PC production is in China.

China's economic development provides local residents with broader employment options, and younger people are increasingly hesitant to work in this industry, and the turnover rate at assembly plants is high. Manufacturers thus have trouble maintain an experienced labor force, resulting in increasingly severe quality issues. To address these problems, manufacturers are increasingly turning to intelligent automation.

This study explores the notebook PC assembly industry, which is characterized by higher output values, growth rates and market share in the $3 \mathrm{C}$ assembly sector. Personal visits, an expert questionnaire and secondary data research were used to reflect the demand for intelligent automation among Taiwan's assembly manufacturers of notebook PCs. The results indicate that investments in intelligent automation must be based on input-output benefits. To this end, manufacturers need to focus on processes characterized by relatively high standardization and high quality thresholds.

\section{Methodology}

Personal visits, an expert questionnaire and secondary data research were used to assess the demand among Taiwan's notebook PC assembly manufacturers for intelligent automation. 


\section{Personal visits}

Researchers visited four international manufacturers (Epson Taiwan, Yaskawa Electric Taiwan Corporation, ABB Ltd. and E-CON Automation Co., Ltd.), and six Taiwan SI manufactures (Wisetech, Vicomm, Angleton, Delta Electronics, Gainwin and Afastor) to understand the demand for intelligent automation.

\section{Expert questionnaire}

A questionnaire based on the Analytic Hierarchy Process (AHP) was completed by 10 domestic experts in intelligent automation to provide information on demand for intelligent automation.

\section{Secondary data}

Information related to the notebook PC assembly industry was taken from domestic and foreign secondary data sources to complement the coverage and integrity of primary data sources. Secondary sources included media reports, special reports, corporate websites, databases of industrial reports and the academic literature.

\section{Findings}

This study first describes quality issues related to products made in China, and then discusses the resulting demand for intelligent automation among international and Taiwanese notebook PC assembly manufacturers.

\section{Quality defects in products made in China}

Most China-based enterprises face difficulty recruiting and retaining qualified staff, and the resulting lack of operating experience leads to poor output quality. This study is concerned with production issues facing two specific products: (1) circuit board processing of LCD panels for industrial equipment and (2) assembly defects in protective alarm transmitters.

\section{(1) Circuit board processing of LCD panels for industrial equipment}

The circuit boards examined here were produced using a production line featuring automated electronic component welding. Despite being a relatively simple and standardized process, manual inspection and repair is still required for defects including:

Ying-Mei Tai is a Researcher in the Machinery \& System Research Division at Industrial Economics and Knowledge Center, also a Ph.D Candidate in Dept. of Business Administration in School of Management at National Yunlin University of Science and Technology (Taiwan). Her Research Topics Focus on Machine Tools, Plastic Machinery, Automobiles, Bicycle, Electric Vehicles, and so on
- Short circuits between electronic components, mainly caused by excessive soldering, and circuit interruption caused by incorrect component installation;

- $\quad$ abnormalities caused by contact between adjacent IC pins which become bent due to excessive heating during welding; and

- $\quad$ IC pin tip-off and short circuits caused by excessive heating.

(2) Assembly defects in protective alarm transmitters

These alarms are used in the prevention of burglaries and to alert parents if children wander off. Defects include too little soldering, missing welds, and warping of the back cover due to failure to remove excess board-materials, thus blocking screw holes and preventing case closure.

These quality issues in China-based manufacturing result from poor SOP compliance and lack of operational experience. These issues will increase in severity as labor force problems continue to worsen, prompting manufacturers to seek alternatives.

Intelligent automation solutions for international assembly manufacturers of notebook PCs

This study is based on international benchmark cases, specifically Panasonic's Kobe plant and Fujitsu's Shimane plant:

\section{(1) Panasonic's Kobe plant}

The plant in Kobe manufactures Panasonic's "Let's Note" and "TOUGHBOOK" notebook PCs. Its manufacturing procedures are divided into motherboard manufacturing and general assembly. The former is highly automatic, with only the Function Board Test (FTB) step still being manually performed and is attempting a robot-based solution. However, the latter still relies on manual operation as a result of great variance.

\section{(2) Fujitsu's Shimane plant}

The plant in Shimane localizes development and manufacture in Japan. Each production line requires only 16 workers, whereas similar production lines in China require an average of 120 workers. To compensate for the cost advantage of China-based manufacturing, the company plans to further improve manufacturing efficiency at Shimane through installing industrial robots.

International notebook PC manufacturers are actively seeking intelligent automation solutions by focusing on processes which are relatively standardized and have high quality thresholds. Processes characterized by high degrees of variance still rely on manual operation because intelligent automation is most cost effective in standardized operations. 
Demand for intelligent automation among Taiwan's notebook PC assembly manufacturers

Most of Taiwan's notebook PC assembly manufactures specialize in OEMs and ODM for international brands. Limited by the small-volume, large-variety production model, manufactures set up plants in China to exploit low-cost labor rather than to actively develop automation solutions. Staffed with tens of thousands and even hundreds of thousands of workers, most large-scale plants usually improve process efficiency only through standardization and simplification.

In this study, the assembly processes for notebook PCs are divided into 5 parts (i.e. motherboard processing, LCD panel processing, palm rest processing, main unit assembly and main unit testing), which are then subdivided into 23 steps (including SMT, inspection and board cutting). Analytic Hierarchy Process (AHP) analysis found that motherboard processing and LCD panel processing are given top priority for intelligent automation ( $25.1 \%$ and $23.9 \%$, respectively), followed by the aging test $(9.4 \%)$, LCD panel assembly $(8.6 \%)$ and functional test ( $8.2 \%$ ) steps (see Fig. 1 for details).

\section{Conclusion}

China-based manufacturing is subject to significant quality issues due to, among other things, poor SOP compliance and lack of operating experience. Continued worsening of workforce issues may exacerbate these quality issues, prompting manufacturers to seek alternatives.

This study explored the notebook PCs assembly industry, which is characterized by higher output value, growth rates and market share in the $3 \mathrm{C}$ assembly sector. Investments in intelligent automation must be based on input-output benefits. Thus, the potential benefits of intelligent automation can only be maximized by selecting relatively standardized and high quality threshold processes.

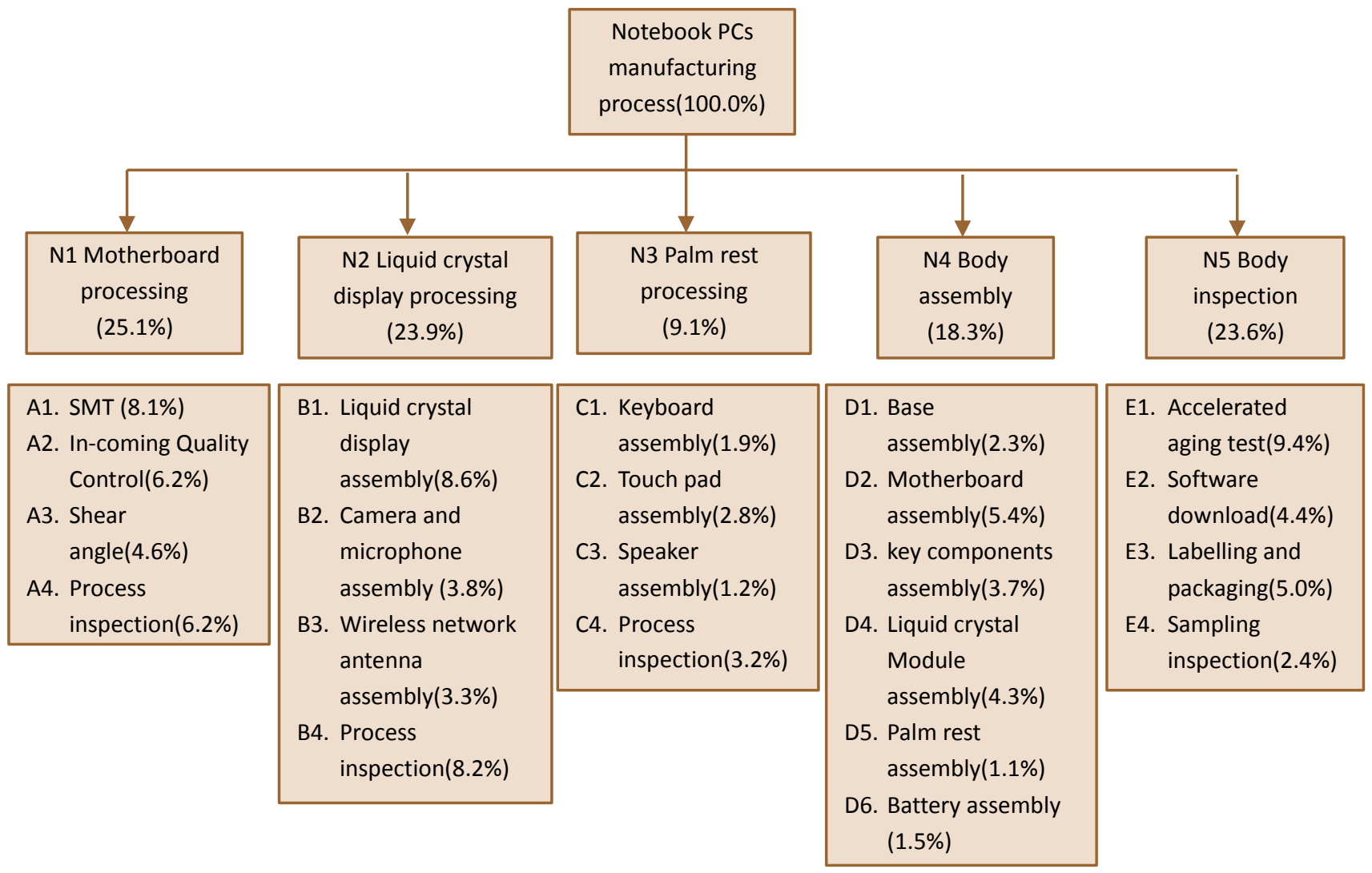

Source: IEK, Industrial Technology Research Institute (Nov. 2012)

Figure 1. Comparison of intelligent automation demands of Notebook PCs assembly manufacturers in Taiwan. 